\title{
A Web of Science-based scientometric analysis about mammalian target of rapamycin signaling pathway in kidney disease from 1986 to 2020
}

\author{
Lian-Zhong Wu ${ }^{1,2 \#}$, Yi-Qin Weng ${ }^{1,2 \#}$, Yi-Xin Ling ${ }^{1,2}$, Shu-Juan Zhou ${ }^{1}$, Xiao-Kai Ding ${ }^{3}$, Si-Qi Wu ${ }^{2}$, \\ Kang $\mathrm{Yu}^{1}$, Song-Fu Jiang ${ }^{1}$, Yi Chen ${ }^{1}$ \\ ${ }^{1}$ Department of Hematology, The First Affiliated Hospital of Wenzhou Medical University, Wenzhou, China; ${ }^{2}$ Wenzhou Medical University, \\ Wenzhou, China; ${ }^{3}$ Department of Nephrology, The First Affiliated Hospital of Wenzhou Medical University, Wenzhou, China \\ Contributions: (I) Conception and design: LZ Wu, YQ Weng; (II) Administrative support: K Yu, SF Jiang; (III) Provision of study materials or \\ patients: None; (IV) Collection and assembly of data: YX Ling, Y Chen; (V) Data analysis and interpretation: SJ Zhou, XK Ding, SQ Wu; (VI) \\ Manuscript writing: All authors; (VII) Final approval of manuscript: All authors. \\ \#These authors contributed equally to this work. \\ Correspondence to: Dr. Yi Chen; Professor Song-Fu Jiang. Department of Hematology, The First Affiliated Hospital of Wenzhou Medical University, \\ Southern Baixiang Road, Ouhai District, Wenzhou 325000, China. Email: 469366150@qq.com; jiangsongfu_1@126.com.
}

\begin{abstract}
Background: The mammalian target of rapamycin (mTOR) signaling pathway is vital for the regulation of cell metabolism, growth and proliferation in the kidney. This study aims to show current research focuses and predict future trends about mTOR pathway in kidney disease by the methods of scientometric analysis.

Methods: We referred to publications from the Web of Science ${ }^{\mathrm{TM}}$ Core Collection (WoSCC) Database. Carrot2, VOSviewer and CiteSpace programs were applied to evaluate the distribution and contribution of authors, institutes and countries/regions of extensive bibliographic metadata, show current research focuses and predict future trends in kidney disease's area.

Results: Until July 10, 2020, there are 2,585 manuscripts about mTOR signaling pathway in kidney disease in total and every manuscript is cited 27.39 times on average. The big name of course is the United States. Research hot spots include "diabetic nephropathy", "kidney transplantation", "autosomal dominant polycystic kidney disease", "tuberous sclerosis complex", "renal cell carcinoma” and "autophagy". Seven key clusters are detected, including "kidney transplantation", "autosomal dominant polycystic kidney disease", "renal transplantation", "renal cell carcinoma", "hamartin”, "autophagy" and "tuberous sclerosis complex".

Conclusions: Diabetic nephropathy, kidney transplantation, autosomal dominant polycystic kidney disease, tuberous sclerosis complex, renal cell carcinoma and autophagy are future research hot spots by utilizing scientometric analysis. In the future, it is necessary to research these fields.
\end{abstract}

Keywords: Bibliometrics; kidney diseases; TOR serine-threonine kinases; VOSviewer; CiteSpace

Submitted Dec 01, 2020. Accepted for publication Dec 24, 2020.

doi: $10.21037 /$ tau-20-1469

View this article at: http://dx.doi.org/10.21037/tau-20-1469

\section{Introduction}

The mammalian target of rapamycin is an evolutionarilyconserved serine-threonine kinase that senses and integrates various environmental factors to regulate cell growth, proliferation and metabolism. Since the discovery of rapamycin's primary target molecular mode of action and the functional biology effect, mammalian target of rapamycin (mTOR) has been recognized to permeate many areas of medicine such as obesity $(1,2)$, type 2 diabetes $(3,4)$, genetic disorders (5), non-alcoholic fatty liver disease (6-8), neurological diseases (9-11) and insulin resistance (12). Increasing evidence indicates that mTOR pathway plays a 
significant role in transplantation, homeostasis, metabolism, and regeneration in the kidney (13-16). In addition, it is related to several diseases such as tuberous sclerosis complex (17), polycystic kidney disease (18), acute kidney injury (19), renal cell carcinoma (20), autosomal dominant polycystic kidney disease (21) and glomerular disease (22).

Bibliometric is a new method for summarizing the statistical analysis of the publications in a specific discipline and subject area, and further identifying warm focus in a research field by creating information graphics. Social science and scientometric analysis use several biometric programs including VOSviewer (23), CitNetExplorer, CiteSpace (24), and HistCite. Even though considerable insights have been gained, many remain to be endeavored regarding the field of biomedicine. Schargus et al. identified the most frequently cited papers in dry eye research (25). Sugimoto et al. made an investigation of sex-related factors in medical research between 2008 and 2016 (26). Fedewa et al. investigated the effect of exercise training on $\mathrm{C}$ reactive protein (27). The purpose of this study is to exploit bibliometric methods in order to analyze literatures regarding mTOR signaling pathway in kidney disease. We investigate the contribution of authors, institutions and countries/regions, the evolution of scientific ideas, research sub-themes, and milestone manuscripts in the specific research filed by utilizing VOSviewer, CiteSpace and other tools.

\section{Methods}

\section{Data source and search strategy}

Relevant literatures were extracted from the Web of Science ${ }^{\mathrm{TM}}$ Core Collection (WoSCC) Database (Clarivate Analysis, Boston, USA). We searched publications by exploiting keywords "mTOR or mammalian target of rapamycin" and "kidney" from WoSCC Database on July 10, 2020. No language restrictions were imposed. "Full record and cited references" were downloaded and raw data were transformed into TXT format which allowed for the analyzation of bibliometric tools.

\section{Statistical analysis and presentation}

Distribution and contribution of authors, institutes and countries/regions and research areas of literatures were analyzed, which was retrieved from WoSCC Database. We applied novel scientometric tools, including VOSviewer
(Ludo Waltman, Leiden University of Centre for Science and Technology Studies, Netherlands), Carrot2 (Dawid Weiss, Poznan University of Technology, Poland) and CiteSpace (Chaomei Chen, Drexel University, USA) for comprehensive science mapping analysis of extensive bibliographic metadata.

CiteSpace (Version 5.6.R2 64-bit), which served as an indicator of the most active area of the scientific community research, was used to capture keywords with strong citation bursts, analyze the time trends of keywords, recognize cocited authors/references, and develop visualization maps. Topical categories were analyzed by Carrot2. VOSviewer (Version 1.6.11) was utilized for recognizing association among journals, and constructing collaboration networks, which referred to term clustering, countries/regions/ institutions/authors and quotation systems of cited authors/ journals.

A total of 2,585 literatures were extracted from WoSCC Database. According to the definition of CiteSpace, visualization knowledge maps were composed of nodes and links. Different nodes in figures represent elements such as countries/regions and authors, and links between nodes indicate relationships of co-citations or collaboration/ cooccurrence. And publications were analyzed to construct the cluster analysis, co-citation network, dual-map overlays, and the time zone or timeline view. We employed the process of "clustering" of CiteSpace to identify different subtopics among all articles about "mTOR in kidney disease". VOSviewer was utilized to perform journal cocitation density analysis. And "Circles visualization" was created to excerpt significant keywords and reveal relative influence to every keyword by Carrot2.

\section{Results}

\section{Annual publications and trend}

Data is input in the flow diagram (Figure 1). Based on the WoSCC, a total of 2,585 manuscripts are published. The first literature about mTOR which met the search terms was published in 1999. Only after 2005 did the articles on mTOR reach at least a double-digit number annually. And the number of publications was an ever-increasing activity per year. In the past 20 years, the proportion of mTOR pathway in the overall research of kidney showed an upward trend (Figure 2A). In 2019, annual literatures grew to 291 (Figure 2A). These papers have been cited 70,795 times and 


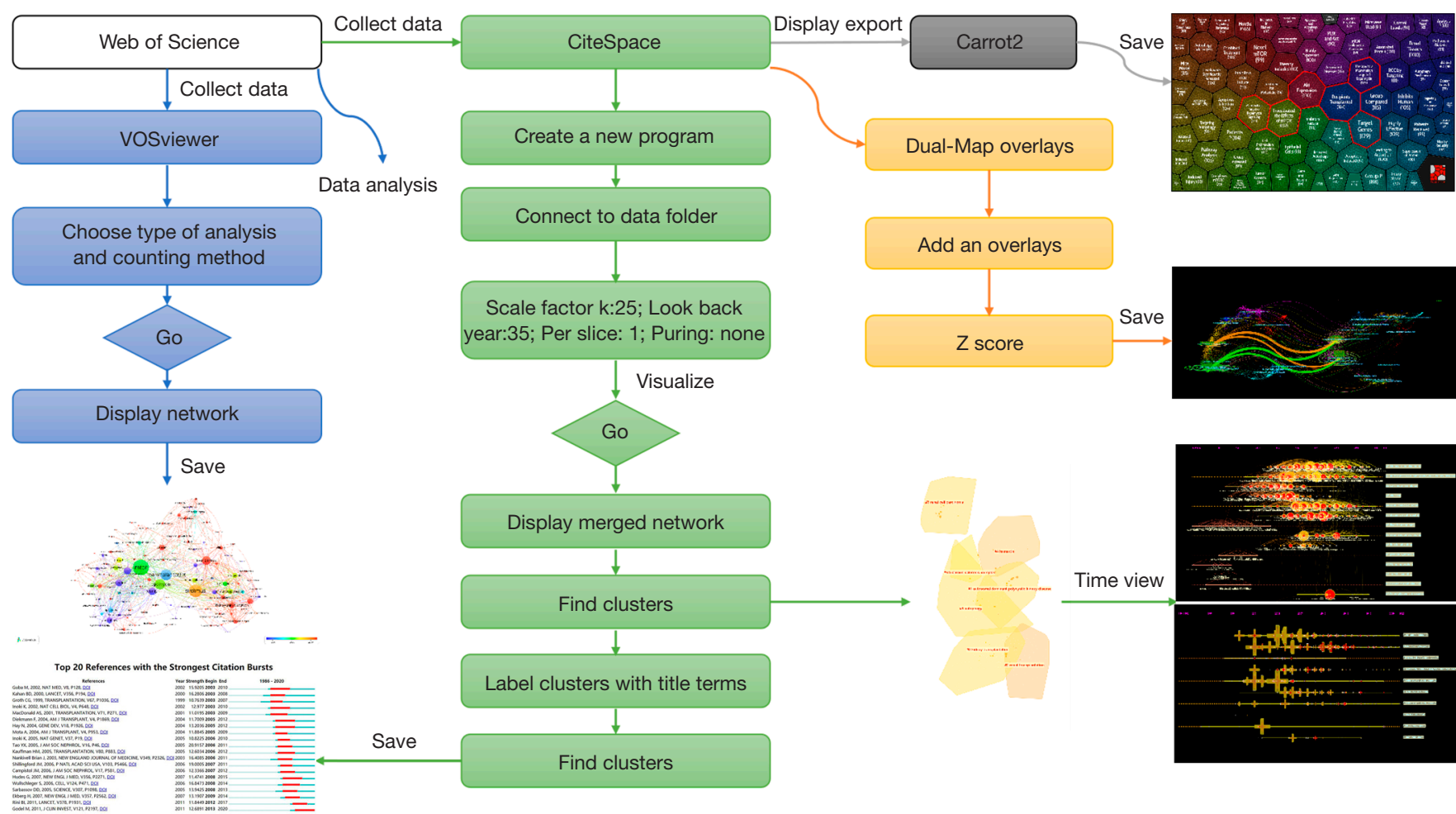

Figure 1 Scientometric analysis of mTOR signaling pathway in kidney disease revealed in the flow diagram. K, scale factor; Z, z-sore, standard score of the quantity of appearances of keywords. mTOR, mammalian target of rapamycin.

every article was averagely cited 27.39 times.

\section{Distribution and contribution of global countries/regions and institutions}

Seventy countries/regions in total have published researches on mTOR signaling pathway in kidney disease. It happened that one literature was published by authors from diverse countries/regions. The top 15 most productive countries/ regions produced 2,860 papers (Figure 2B). Papers about mTOR in kidney disease originated mainly in the United States. And the number of papers in the United States was a lot higher than in the other countries/regions. The USA published 923 articles, followed by China ( $\mathrm{n}=373)$, Germany $(\mathrm{n}=297)$, Italy $(\mathrm{n}=199)$, France $(\mathrm{n}=194)$, Spain $(\mathrm{n}=181)$, Japan $(\mathrm{n}=126)$, England $(\mathrm{n}=108)$ and Switzerland $(\mathrm{n}=94)$ (Figure $2 B)$. According to the heat map made by VOSviewer, the USA, Germany, Italy and China had the most intense publication density (Figure 2C).

In order to obtain a better understanding of the contributions of each country/region to the mTOR in kidney disease and the collaborations between countries/ regions, we analyzed the WoSCC Database as data. According to these data, we focused more on international collaborations, the number of citations, and the times each country/region cites the work of another. USA $(n=32,984)$ and Germany $(\mathrm{n}=8,579)$ ranked top two regard to total citation numbers (Figure 2D). Although the USA, China, Germany, Italy and France have the highest numbers of mTOR-related publications, the average citation number per document of Switzerland and Netherlands is much higher than the other countries (Figure 2D). China, Japan and some European nations, such as Germany, England and Netherlands, had the largest publications concentrated between 2008 and 2010, while Italy, Poland, Canada, Switzerland and Spain are mainly published from 2013 to 2015 (Figure 2E).

A total of 2,882 institutions have published literatures. The citation network among these institutions was analyzed by VOSviewer program. Table 1 revealed the top 10 institutions with the most publications. Among these organizations, five belong to the United State, two to Germany, two to Spain and one to France. The Harvard University mainly researched mTOR in kidney disease 
A

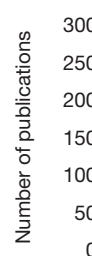

300

250

200

150

100

50

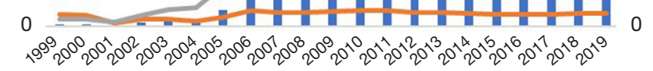

Year

A=Number of publications ("mTOR or mammalian target of rapamycin" and "kidney")

[A/Number of publications (mTOR or mammalian target of rapamycin)] $\times 100$

- [AVNumber of publications (kidney)] $\times 10000$

C

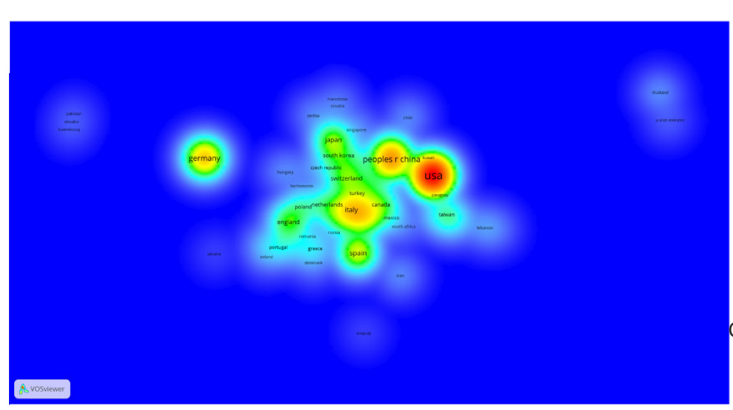

$\mathrm{E}$
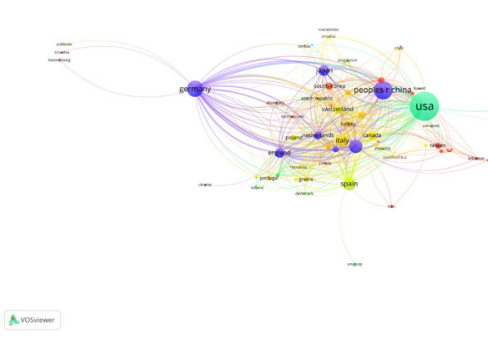

G

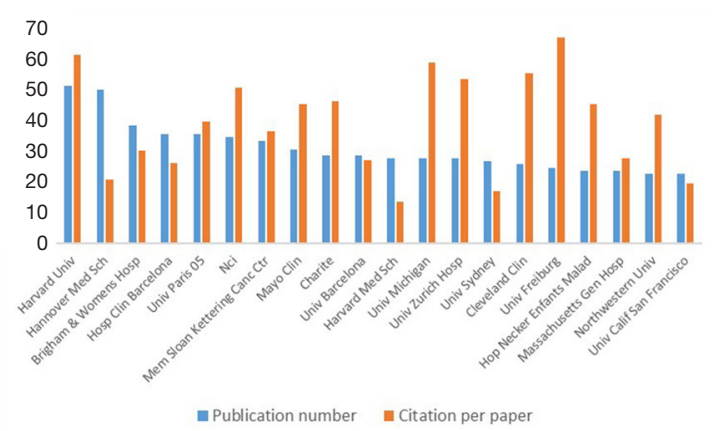

B

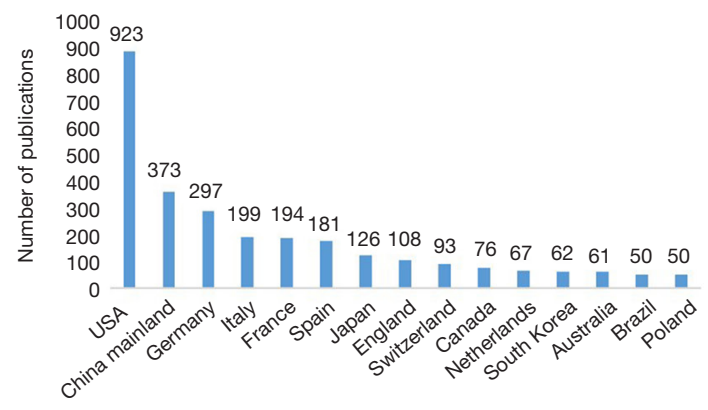

$D$

Poland -15.26 .52$

Brazil $={ }_{5.72}^{11.44}$

South Korea ${ }_{7.26} 6^{19}$

Netherlands $\quad 27.27^{40.70}$

Canada $=28.38 .68$

Switzerland $\quad{ }_{44.47}$

England $={ }_{41.29}^{38.23}$

Japan $\quad 266_{32.9}^{49}$

Spain $\quad 20.4937 .09$

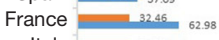

Italy $\quad 3175$

Germany 8579

China mainland $=15.69$

USA

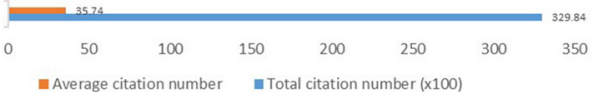

$\mathrm{F}$

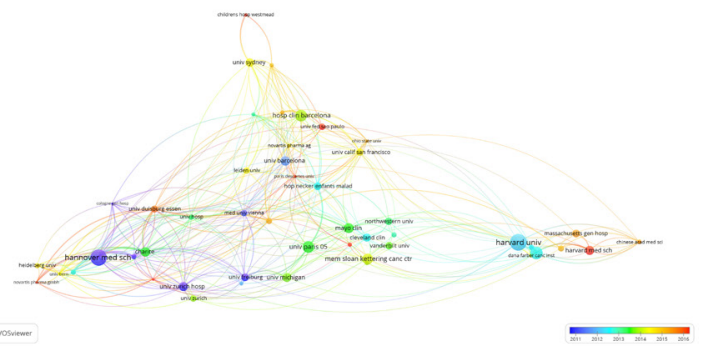

$\mathrm{H}$

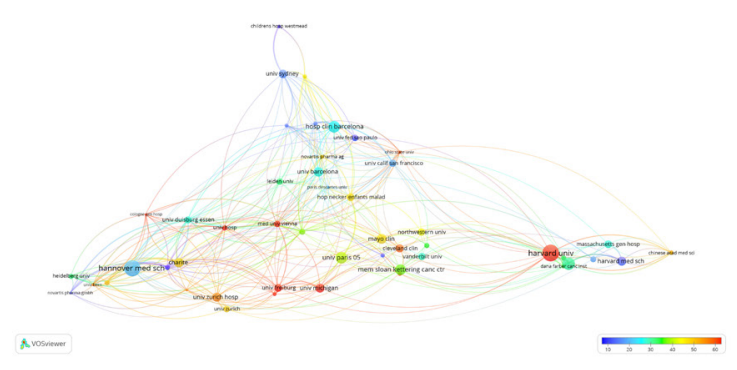

Figure 2 Countries/regions involved in mTOR signaling pathway in kidney disease research. (A) Number and percentage of publications. (B) The top 15 countries/regions with the largest publications. (C) Heat map of publications in countries/regions. (D) Average citations per paper and total citation number of top 15 countries/regions with the largest production. (E) The number of articles in 70 countries/ regions. (F) The number of manuscripts in different institutions. (G) Publication number and citation per paper of top 20 most productive institutions. $(\mathrm{H})$ Collaboration between institutions. mTOR, mammalian target of rapamycin. 
Table 1 The top 15 institutes ranked by the citation number of articles about mTOR signaling pathway in kidney disease

\begin{tabular}{|c|c|c|c|c|c|}
\hline Rank & Institution & Country/region & Citations & Publications & Average citation per paper \\
\hline 2 & $\mathrm{NCl}$ & USA & 1,803 & 35 & 51.51 \\
\hline 3 & Univ Freiburg & Germany & 1,704 & 25 & 68.16 \\
\hline 4 & Univ Michigan & USA & 1,675 & 28 & 59.82 \\
\hline 6 & Univ Zurich Hosp & Switzerland & 1,523 & 28 & 54.39 \\
\hline 7 & Cleveland Clin & USA & 1,464 & 26 & 56.31 \\
\hline 8 & Univ Paris 05 & France & 1,447 & 36 & 40.19 \\
\hline 9 & Mayo Clin & USA & 1,428 & 31 & 46.06 \\
\hline 12 & Mem Sloan Kettering Canc Ctr & USA & 1,258 & 34 & 37.00 \\
\hline 13 & Brigham \& Womens Hosp & USA & 1,194 & 39 & 30.62 \\
\hline 14 & Hop Necker Enfants Malad & France & 1,109 & 24 & 46.21 \\
\hline 15 & Hannover Med Schm & Germany & 1,073 & 51 & 21.04 \\
\hline
\end{tabular}

TOR, mammalian target of rapamycin.

in 2012-2013 (Figure 2F). On average, papers from the University Freiburg were cited 68.16 times, which was much higher than the others (Figure 2G,H).

\section{Manuscript distribution among journals}

The 2,585 articles were extracted from 730 SCI-E recorded journals. The total publication number and the citation network among these journals were analyzed by VOSviewer program. The minimum number of documents of journals was set higher than 5 to visualize a map of 100 journals (Figure 3A). The top 20 most productive journals published 850 literatures, which was one out of three of the total publication number. The top three magazines with the most evaluated numbers of distributed articles were Transplantation Proceedings, Transplantation, and American fournal of Transplantation. Eighteen of these journals were established in the UK $(\mathrm{n}=5)$ and the United States ( $\mathrm{n}=13)$ (Table 2). New England Fournal of Medicine (74.699), Nature Medicine (36.13), Nature Reviews Nephrology (20.711), Annals of Oncology (18.274), and European Urology (17.947) were the top 5 journals with the highest impact factor. Since Nature had only published one related literature: termination of autophagy and reformation of lysosomes regulated by mTOR (28), even if the citation number reached 783 , the VOSviewer program did not select the magazine. The same research area of journals had a tendency to be within the same cluster. The node on the graph represents a magazine and links between nodes indicate co-citation relationship with each other (Figure 3A). There existed three clusters in the network visualization map and items in different clusters have different colors (Figure 3B).

\section{Review of milestone authors and literatures}

A total of 14,045 researchers had published the 2,585 manuscripts and the number of authors every paper on average was 5.43. We used CiteSpace and VOSviewer to analyze coauthorship and citation network among authors and to reveal the most highly cited researchers and collaboration among researchers. "Key researcher" was referred to as someone whose literatures had been cited more than 500 times (global citation score $>500$ ). The nodes indicated different researchers. Lines between nodes represented the cooperation between authors, and the size of the node showed the number of papers published by the author (Figure 3C,D). Linehan WM, Schmidt LS, Sommerer C, 
A

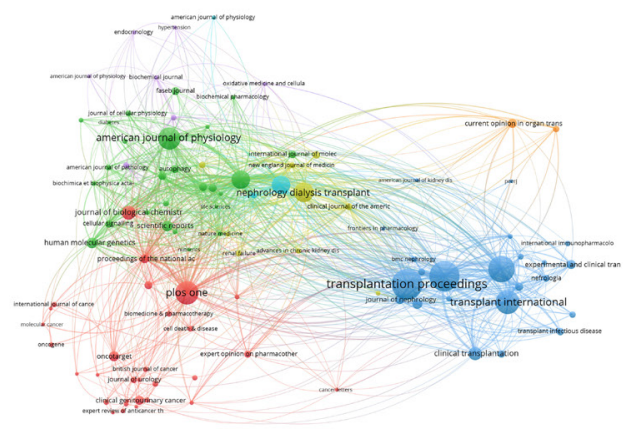

C

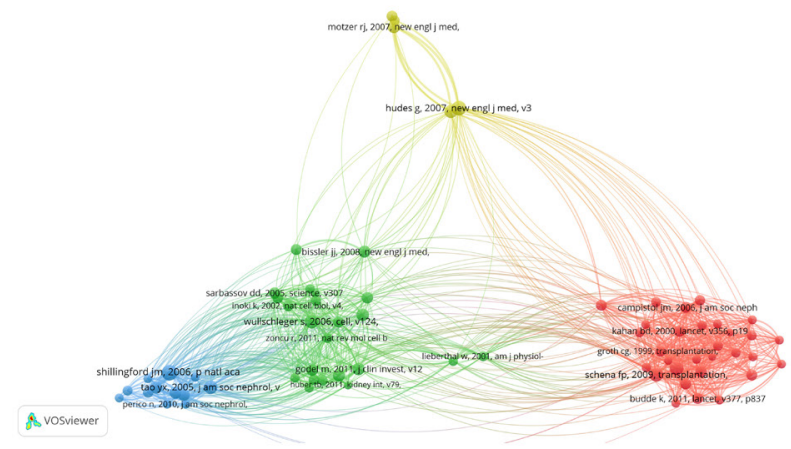

$E$

Top 20 References with the Strongest Citation Bursts

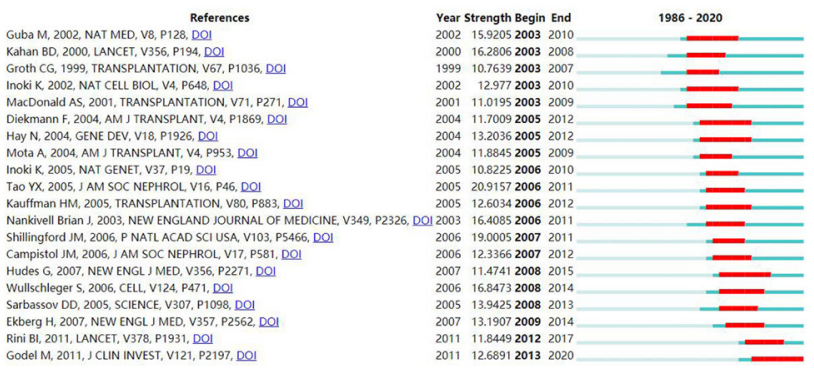

B

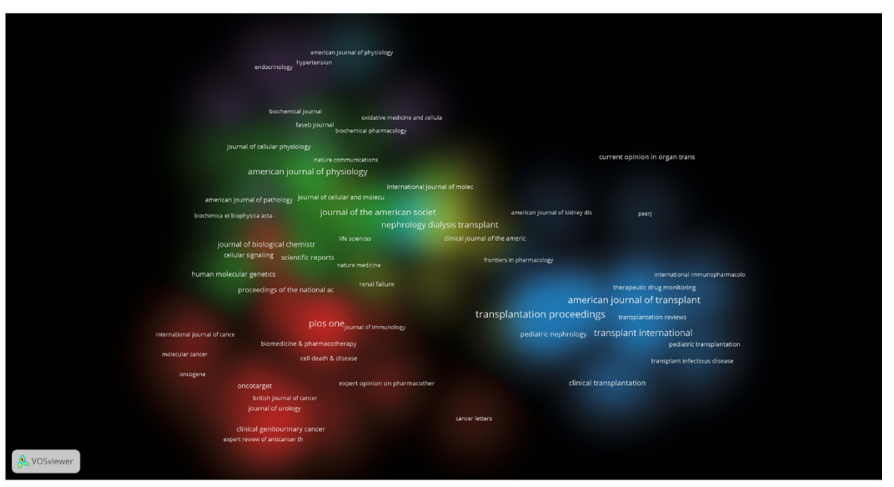

D
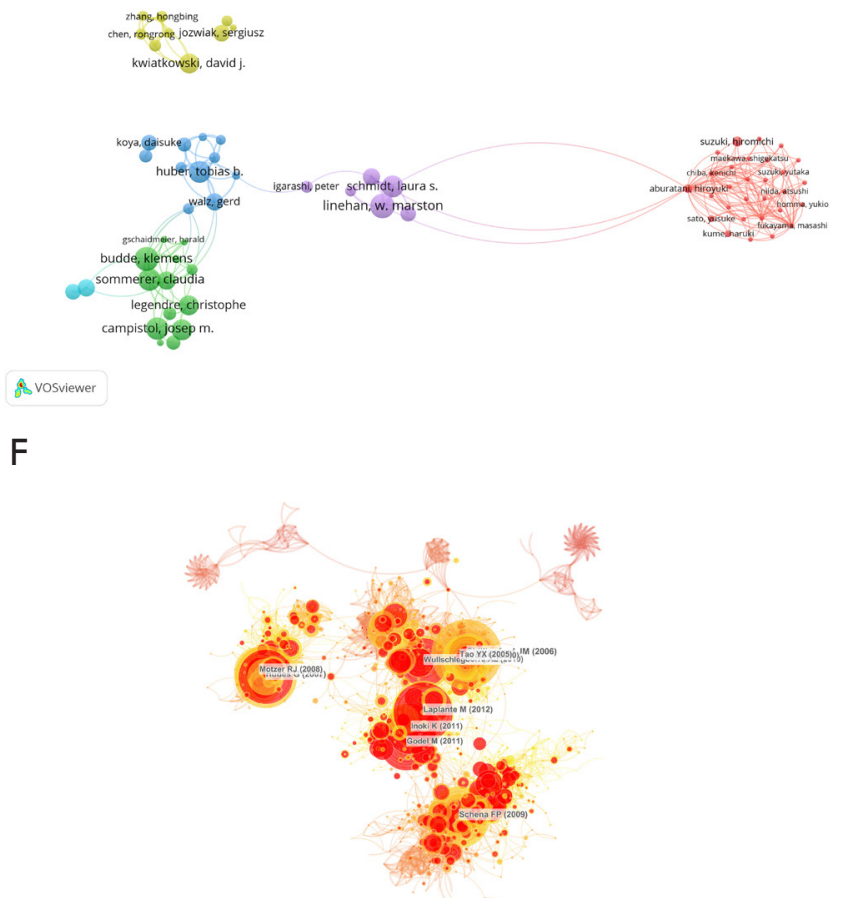

Figure 3 Citation networks and collaboration among organizations and authors. (A) The top 100 highly cited journals with network visualization. (B) The top 100 highly cited journals with citation network. (C) The co-authorship among top 70 highly cited authors. (D) The top 70 highly cited authors with network visualization. (E) The top 20 strongest citation bursts of references. (F) A co-citation network of references.

Kasinath BS, Aburatani H and Baba M made outstanding contributions to the research of mTOR signaling pathway in kidney disease. Linehan WM published 24 papers in this research area. Linehan WM, Schmidt LS and Huber $\mathrm{TB}$, who can be considered as the leaders of this research area, each received over 1,000 citations. Linehan WM and Aburatani $\mathrm{H}$ collaborated on many articles, but their cooperation with Kwiatkowski DJ was not as frequent (Figure 3D). The 2,585 literatures about mTOR signaling pathway in kidney disease also cited each other or a few other studies in their references. We utilized CiteSpace to analyze 71,544 references of their compositions and produce the top 20 strongest citation bursts and a streamlined cocitation network (Figure 3E,F). The figure for citations 
Table 2 The top 20 journals contributed to mTOR signaling pathway in kidney disease ranked by citation number

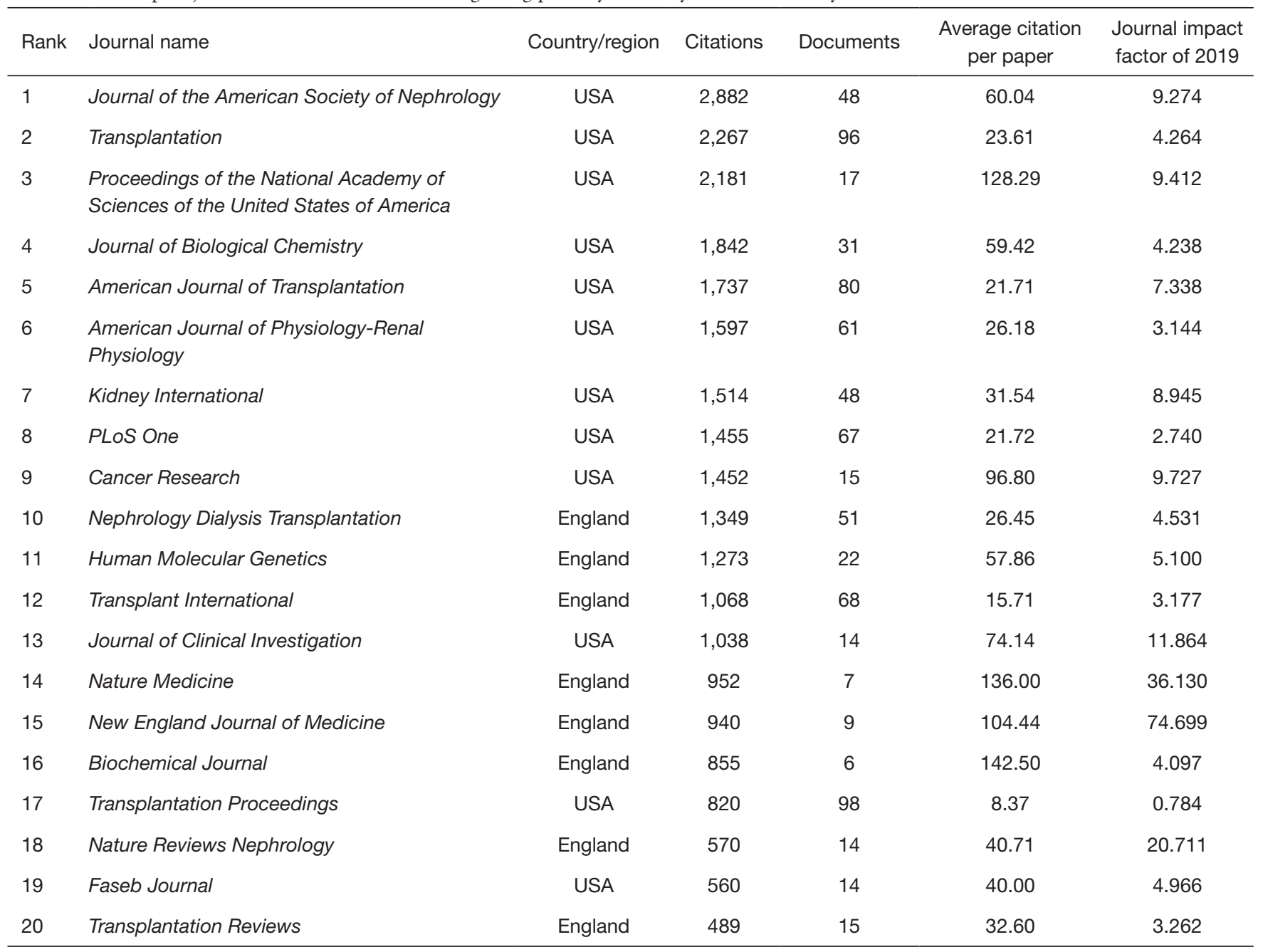

of these publications suddenly boosted during a span of time, which revealed the rapid acceptance and spreading in specific ground of research and occupied very important positions in the knowledge frame.

\section{Keywords and MeSH terms visualization}

The occurrence frequency and time trend of keywords and $\mathrm{MeSH}$ terms were visualized by the program of CiteSpace and VOSviewer. In order to extract key concepts of the mTOR in kidney disease, we used the Carrot 2 system to analyze major topics. There were top 6 keywords in the red frame, which represented the highest rate of occurrence with "Recipients Transplanted", "Investigated the Effects of mTOR”, “Akt Expression”, "Target Genes”, "Mammalian
Target of Rapamycin Signaling" and "Therapy to a Mammalian Target of Rapamycin" (Figure 4A). On the basis of CiteSpace, the co-citation network was segmented into different diverse clusters from the titles of the cited articles (Figure 4B). Network visualization of keywords and clustering survey of mTOR were based on VOSviewer program (Figure 4C,D).

The software of CiteSpace was utilized to analyze time zone view and timeline view of mTOR signaling pathway in kidney disease co-citation network. The time of publication was revealed at the top of the view. The figure for nodes in a horizontal line represented the importance of study field and the link between nodes reflected citing. The timeline view also showed the occurrence, popularity, and decline of research subjects in the most typical publications. 
A

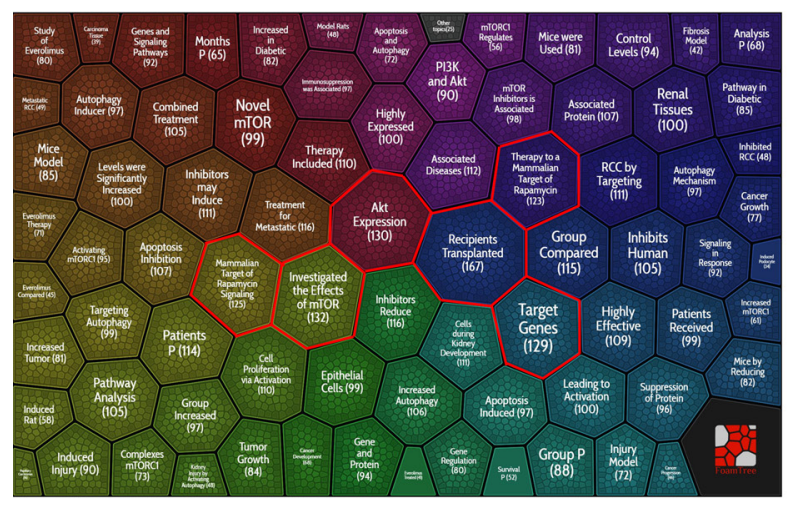

C

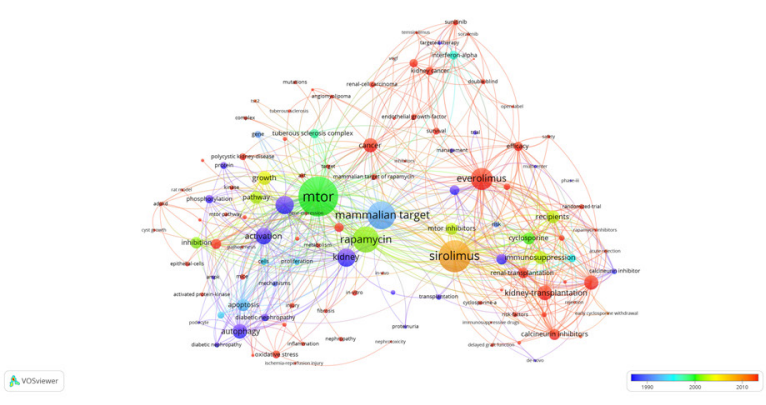

B
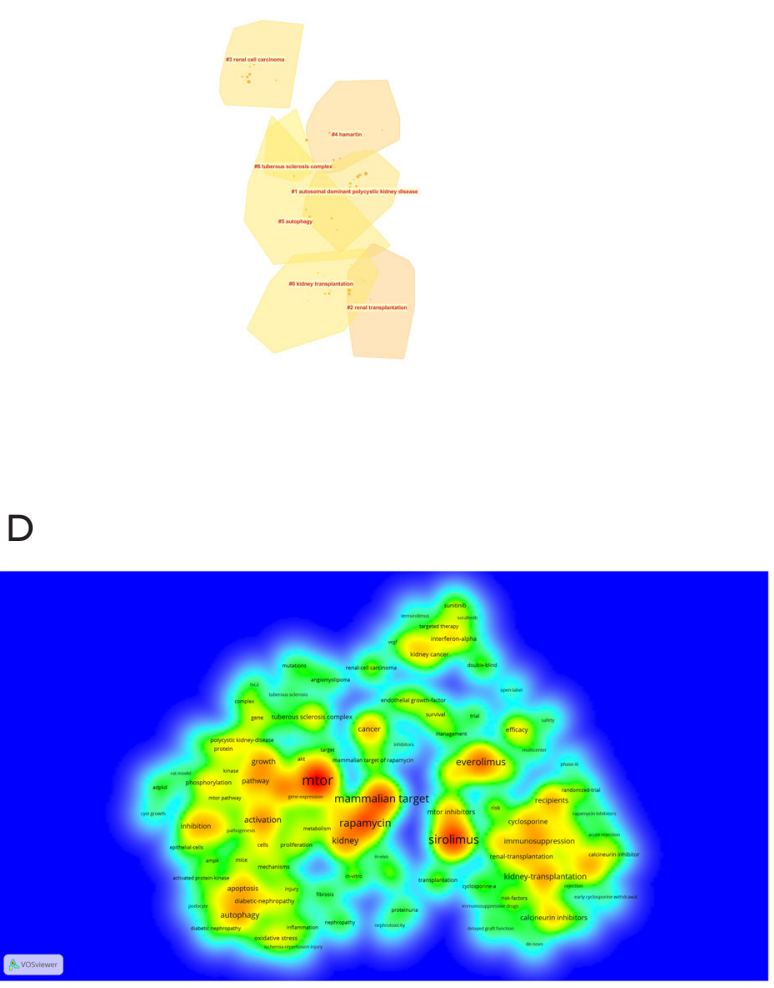

Figure 4 The visualization of keywords and MeSH terms. (A) The keywords analysis for the mTOR signaling pathway in kidney disease. (B) The clustering analysis of co-citation network for the mTOR signaling pathway in kidney disease. (C) The network visualization of keywords. (D) Heat map of keywords.

Meanwhile, clusters reflected the temporal characteristics of the research areas in timeline view. The development of cluster 10 (binding protein) showed the early exploration of the mTOR pathway in kidney research focusing on binding protein, which occurred earliest. Cluster 0 (kidney transplantation), cluster 1 (autosomal dominant polycystic kidney disease), cluster 4 (renal cell carcinoma), cluster 5 (diabetic nephropathy), cluster 7 (renal angiomyolipoma) and cluster 13 (non-glycemic diseases) were current research frontiers and focuses (Figure $5 A$ ). The analysis results of keywords timeline revealed calcineurin inhibitor, diabetic nephropathy, autosomal dominant polycystic kidney disease and tuberous sclerosis complex (Figure 5B). Current research focuses on mechanism and complications of kidney disease. The time zone view of cluster nodes illustrated when the co-citation network cited publications, which could clearly demonstrate the research progress over time as well (Figure 5C). And the central connection between time zones revealed the extent of which message was missing or message was acknowledged so as to stimulate revelations and promote investigations. If there were more literatures in a time zone visualization, it would be a significant period (Figure 5C).

Dual-map analysis was utilized to indicate generate scientific connections and contributions. The citing outline is on the left half and the cited outline is on the right half. The quotation association line in trajectory from one region to another would illustrate comprehension of inter-specialty relationships, thereby driving connections and knowledge of different research fields (Figure 5D).

\section{Discussion}

Different from traditional reviews, bibliometric analysis can systematically process the manuscripts in related research areas in order to get more intuitive and clear results. For 
A

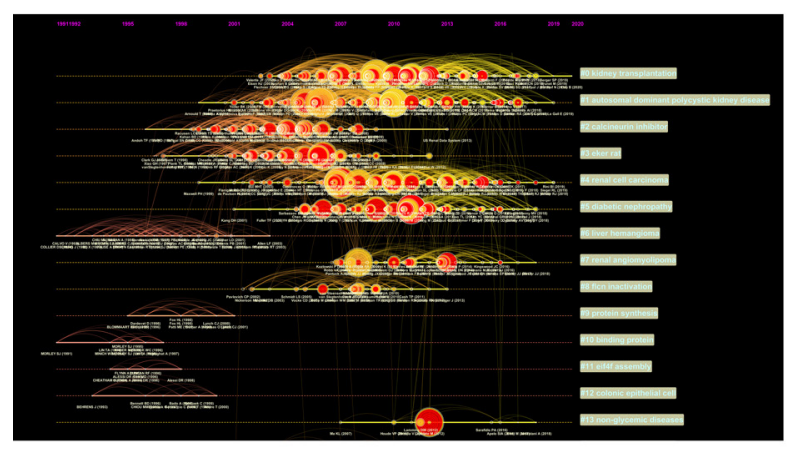

C

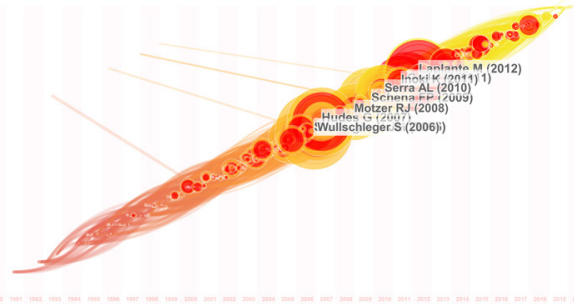

B

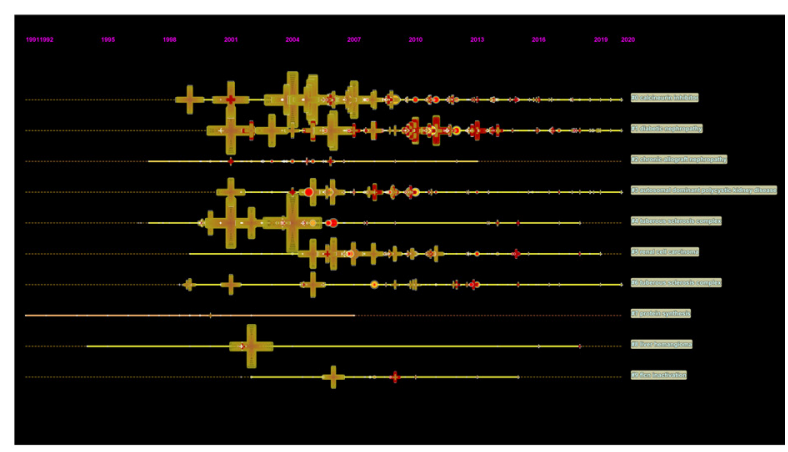

D

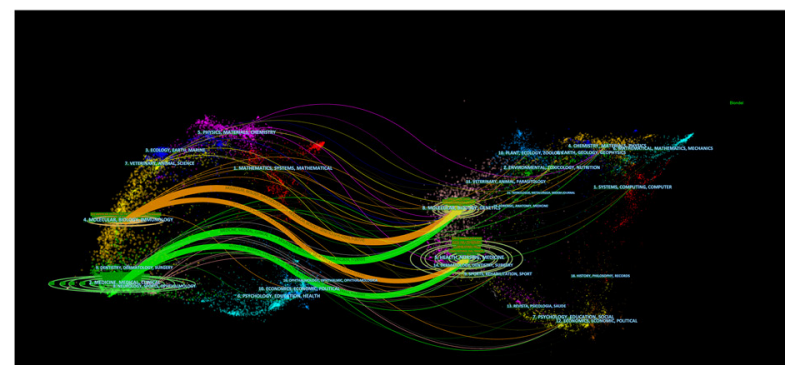

Figure 5 Time zone view of cluster nodes and timeline visualization of keywords and MeSH terms. (A) The visualization of term timeline from 1986 to 2020. (B) The visualization of keywords timeline from 1986 to 2020. (C) The visualization of time zone view of cluster nodes. (D) Dual-map overlays of publications about mTOR in kidney disease.

new researchers to specific areas, it can be helpful to follow the current study focuses and overall trends and reveal landmark literatures in the field. Furthermore, it is easy and user-friendly to operate current bibliometrics analysis software. In current research, we presented a bibliometric analysis regarding the mTOR signaling pathway in kidney disease.

On the basis of the results, a total of 2,585 literatures with 71,544 references were extracted from WoSCC. The figure for manuscripts is increasing per year. Although the mTOR signaling pathway is a branch of the overall research of kidney, its research trend is has gradually increased recently (Figure 2A). The USA, China, and Germany are more prominent in this field, accounting for $55.7 \%$ of the publications. Five of the top 20 most productive institutions are in the United States (Table 1), which shows that the quality of literatures published by American institutions is higher. Research institutions, such as Harvard University (Figure $2 F, G, H$ ), are relatively mature in this specific area and can be regarded as a significant institution for learning and further collaboration. These results indicate that the USA occupies its leading position in mTOR signaling pathway in kidney disease, mainly reflecting in international collaborations, total citations and publication numbers. Notably, compared with Germany and England, publications of other European countries such as Italy and Switzerland, have increased dramatically after 2014, revealing that more developed countries/regions will devote to further investigation in this area (Figure 2E).

Journals in the same cluster possess the same area of research. Using the function of VOSviewer software, we divided the top 100 journals into three clusters (Figure 3B). Based on CiteSpace, the entire research fields were detected and divided fourteen subsets and the largest seven clusters are on display in Figure 4B, including cluster 0 "kidney transplantation", cluster 1 "autosomal dominant polycystic kidney disease", cluster 2 "renal transplantation", cluster 3 "renal cell carcinoma", cluster 4 "hamartin", cluster 5 "autophagy" and cluster 6 "tuberous sclerosis complex". Cluster 3 "renal cell carcinoma" indicate that mTOR is significant in the research of renal cell carcinoma (RCC) and the antiproliferative property of mTOR inhibitors may suppress tumor growth. RCC ranks seventh and ninth among men and women, respectively, and numbers of new cancer 
cases will be increased in the future (29). Surgical resection is the main method for the treatment of tumors that are confined to the kidney. About one third of patients have distant metastasis, and most cases are incurable. Interferon is widely used in translocation renal cell carcinoma, but its efficacy and tolerance are limited. Therefore, new therapeutic targets of mTOR come into focus $(30,31)$. We used CiteSpace to carry out co-citation analysis and determined several milestone literatures (Figure $3 F$ ). For example, Linehan WM revealed the genetic basis of RCC and the role of mTOR pathway in its pathogenesis (32). Hudes et al. also assessed temsirolimus a mTOR inhibitor for RCC (33). Serra et al. evaluated sirolimus and kidney growth in autosomal dominant polycystic kidney disease (34). The work by Laplante $e t$ al. has the biggest citation burst (Figure $3 F$ ): mTOR signaling in growth control and disease (4). Shillingford et al. elaborated that the mTOR pathway inhibition could reverse renal cystogenesis in polycystic kidney disease (35). The manuscript was included in cluster 1 "autosomal dominant polycystic kidney disease" and cluster 5 "autophagy".

Readers can authoritatively recognize the mechanism of mTOR in kidney transplantation, kidney cancer and renal tuberous sclerosis complex, and their treatment of kidney disease through these milestone manuscripts. According to Figure $5 A, B$, the development of the mTOR in kidney disease over specific time is showed clearly. Calcineurin inhibitor is a research hotspot and researches have illustrated the comparison of calcineurin inhibitors and mTOR inhibitors to treat kidney disease $(13,36,37)$. In addition, the research of the mTOR signaling pathway in kidney disease might begin related to mTOR (38-40). We utilized VOSviewer and Carrot2 to recognize keywords of importance (Figure 4A,C,D). Among the hotpots in Figure 4C,D, there are multiple names of drugs, such as rapamycin, sirolimus and everolimus and so on. In 1999, the US Food and Drug Administration (FDA) approved the marketing of rapamycin as a new treatment option to prevent organ rejection in renal transplant recipients (41). Therefore, research on rapamycin is mainly concentrated around 2000 (Figure 4C). In March 2010, everolimus has obtained the US FDA and the European Medicines Agency approval for the treatment of advanced RCC and renal transplantation (42). And thus, the research of everolimus and renal transplantation mostly focused on 2010 (Figure 4C). We analyzed time trends of keywords by CiteSpace (Figure 5A,B,C). Diabetic nephropathy, kidney transplantation, autosomal dominant polycystic kidney disease, tuberous sclerosis complex, renal cell carcinoma and autophagy might be research focuses over the next few years.

According to dual-map overlays (Figure 5D), the citation trajectory tells us that medicine and biology are the dominant regions of mTOR signaling pathway in kidney disease, and mTOR has a little relation with other disciplines. Chiew et al. have portrayed a three-dimensional platform by using fluorescence resonance energy transferbased caspase- 3 sensor to detect the apoptotic capability of mTOR inhibitors (43). With further research about the mTOR signaling in kidney disease, other subjects will eventually be involved. However, this study has some limitations, which must be considered when analyzing the results of researches. Firstly, the literatures are only extracted from WoSCC Database, which could result in incomplete document retrieval. Secondly, we have detected some clusters, such as liver hemangioma and colonic epithelial cell, which are not closely related to kidney disease.

\section{Conclusions}

Future research hotspots will embody in diabetic nephropathy, kidney transplantation, autosomal dominant polycystic kidney disease, tuberous sclerosis complex, renal cell carcinoma and autophagy. Based on various scientific methods, it can provide future research direction for those who are new to enter this area.

\section{Acknowledgments}

Funding: This work was supported by the National Natural Science Foundation of China (grant number 81703851).

\section{Footnote}

Peer Review File: Available at http://dx.doi.org/10.21037/ tau-20-1469

Conflicts of Interest: All authors have completed the ICMJE uniform disclosure form (available at http://dx.doi. org/10.21037/tau-20-1469). The authors have no conflicts of interest to declare.

Ethical Statement: The authors are accountable for all aspects of the work in ensuring that questions related to the accuracy or integrity of any part of the work are appropriately investigated and resolved. 
Open Access Statement: This is an Open Access article distributed in accordance with the Creative Commons Attribution-NonCommercial-NoDerivs 4.0 International License (CC BY-NC-ND 4.0), which permits the noncommercial replication and distribution of the article with the strict proviso that no changes or edits are made and the original work is properly cited (including links to both the formal publication through the relevant DOI and the license). See: https://creativecommons.org/licenses/by-nc-nd/4.0/.

\section{References}

1. Aleman JO, Eusebi LH, Ricciardiello L, et al. Mechanisms of obesity-induced gastrointestinal neoplasia. Gastroenterology 2014;146:357-73.

2. D'Agati VD, Chagnac A, de Vries AP, et al. Obesity-related glomerulopathy: clinical and pathologic characteristics and pathogenesis. Nat Rev Nephrol 2016;12:453-71.

3. Blandino-Rosano M, Barbaresso R, Jimenez-Palomares $\mathrm{M}$, et al. Loss of mTORC1 signalling impairs betacell homeostasis and insulin processing. Nat Commun 2017;8:16014.

4. Laplante $M$, Sabatini DM. mTOR signaling in growth control and disease. Cell 2012;149:274-93.

5. Li J, Kim SG, Blenis J. Rapamycin: one drug, many effects. Cell Metab 2014;19:373-9.

6. Uno K, Yamada T, Ishigaki Y, et al. A hepatic amino acid/ mTOR/S6K-dependent signalling pathway modulates systemic lipid metabolism via neuronal signals. Nat Commun 2015;6:7940.

7. Kim K, Qiang L, Hayden MS, et al. mTORC1independent Raptor prevents hepatic steatosis by stabilizing PHLPP2. Nat Commun 2016;7:10255.

8. Han J, Li E, Chen L, et al. The CREB coactivator CRTC2 controls hepatic lipid metabolism by regulating SREBP1. Nature 2015;524:243-6.

9. Lipton JO, Sahin M. The neurology of mTOR. Neuron 2014;84:275-91.

10. Bockaert J, Marin P. mTOR in Brain Physiology and Pathologies. Physiol Rev 2015;95:1157-87.

11. Crino PB. The mTOR signalling cascade: paving new roads to cure neurological disease. Nat Rev Neurol 2016;12:379-92.

12. Ardestani A, Lupse B, Kido Y, et al. mTORC1 Signaling: A Double-Edged Sword in Diabetic beta Cells. Cell Metab 2018;27:314-31.

13. Fantus D, Rogers NM, Grahammer F, et al. Roles of mTOR complexes in the kidney: implications for renal disease and transplantation. Nat Rev Nephrol 2016;12:587-609.

14. Shiels PG, McGuinness D, Eriksson M, et al. The role of epigenetics in renal ageing. Nat Rev Nephrol 2017;13:471-82.

15. Orhon I, Dupont N, Zaidan M, et al. Primary-ciliumdependent autophagy controls epithelial cell volume in response to fluid flow. Nat Cell Biol 2016;18:657-67.

16. Grahammer F, Nesterov V, Ahmed A, et al. mTORC2 critically regulates renal potassium handling. J Clin Invest 2016;126:1773-82.

17. MacKeigan JP, Krueger DA. Differentiating the mTOR inhibitors everolimus and sirolimus in the treatment of tuberous sclerosis complex. Neuro Oncol 2015;17:1550-9.

18. Menezes LF, Germino GG. The pathobiology of polycystic kidney disease from a metabolic viewpoint. Nat Rev Nephrol 2019;15:735-49.

19. Gui Y, Lu Q, Gu M, et al. Fibroblast mTOR/ PPARgamma/HGF axis protects against tubular cell death and acute kidney injury. Cell Death Differ 2019;26:2774-89.

20. Choueiri TK, Je Y, Sonpavde G, et al. Incidence and risk of treatment-related mortality in cancer patients treated with the mammalian target of rapamycin inhibitors. Ann Oncol 2013;24:2092-7.

21. Gimpel C, Bergmann C, Bockenhauer D, et al. International consensus statement on the diagnosis and management of autosomal dominant polycystic kidney disease in children and young people. Nat Rev Nephrol 2019;15:713-26.

22. Allison SJ. Glomerular disease: mTOR in FSGS. Nat Rev Nephrol 2017;13:260.

23. van Eck NJ, Waltman L. Citation-based clustering of publications using CitNetExplorer and VOSviewer. Scientometrics 2017;111:1053-70.

24. Chen C, Song M. Visualizing a field of research: A methodology of systematic scientometric reviews. PLoS One 2019;14:e223994.

25. Schargus M, Kromer R, Druchkiv V, et al. The top 100 papers in dry eye - A bibliometric analysis. Ocul Surf 2018;16:180-90.

26. Sugimoto CR, Ahn YY, Smith E, et al. Factors affecting sex-related reporting in medical research: a crossdisciplinary bibliometric analysis. Lancet 2019;393:550-9.

27. Fedewa MV, Hathaway ED, Ward-Ritacco CL. Effect of exercise training on $\mathrm{C}$ reactive protein: a systematic review and meta-analysis of randomised and non-randomised controlled trials. Br J Sports Med 2017;51:670-6. 
28. Yu L, McPhee CK, Zheng L, et al. Termination of autophagy and reformation of lysosomes regulated by mTOR. Nature 2010;465:942-6.

29. Siegel RL, Miller KD, Jemal A. Cancer statistics, 2019. CA Cancer J Clin 2019;69:7-34.

30. Haas NB, Appleman LJ, Stein M, et al. Autophagy Inhibition to Augment mTOR Inhibition: a Phase I/II Trial of Everolimus and Hydroxychloroquine in Patients with Previously Treated Renal Cell Carcinoma. Clin Cancer Res 2019;25:2080-7.

31. Xu J, Pham CG, Albanese SK, et al. Mechanistically distinct cancer-associated mTOR activation clusters predict sensitivity to rapamycin. J Clin Invest 2016;126:3526-40.

32. Linehan WM, Schmidt LS, Crooks DR, et al. The Metabolic Basis of Kidney Cancer. Cancer Discov 2019;9:1006-21.

33. Hudes G, Carducci M, Tomczak P, et al. Temsirolimus, interferon alfa, or both for advanced renal-cell carcinoma. N Engl J Med 2007;356:2271-81.

34. Serra AL, Poster D, Kistler AD, et al. Sirolimus and kidney growth in autosomal dominant polycystic kidney disease. N Engl J Med 2010;363:820-9.

35. Shillingford JM, Murcia NS, Larson CH, et al. The mTOR pathway is regulated by polycystin-1, and its inhibition reverses renal cystogenesis in polycystic kidney disease. Proc Natl Acad Sci U S A 2006;103:5466-71.

Cite this article as: Wu LZ, Weng YQ, Ling YX, Zhou SJ, Ding XK, Wu SQ, Yu K, Jiang SF, Chen Y. A Web of Sciencebased scientometric analysis about mammalian target of rapamycin signaling pathway in kidney disease from 1986 to 2020. Transl Androl Urol 2021;10(3):1006-1017. doi: 10.21037/ tau-20-1469
36. Guo Z, Hong SY, Wang J, et al. Rapamycin-inspired macrocycles with new target specificity. Nat Chem 2019;11:254-63.

37. Budde K, Becker T, Arns W, et al. Everolimus-based, calcineurin-inhibitor-free regimen in recipients of de-novo kidney transplants: an open-label, randomised, controlled trial. Lancet 2011;377:837-47.

38. Henske EP, Jozwiak S, Kingswood JC, et al. Tuberous sclerosis complex. Nat Rev Dis Primers 2016;2:16035.

39. Narita M, Young AR, Arakawa S, et al. Spatial coupling of mTOR and autophagy augments secretory phenotypes. Science 2011;332:966-70.

40. Natoli TA, Smith LA, Rogers KA, et al. Inhibition of glucosylceramide accumulation results in effective blockade of polycystic kidney disease in mouse models. Nat Med 2010;16:788-92.

41. Miller JL. Sirolimus approved with renal transplant indication. Am J Health Syst Pharm 1999;56:2177-8.

42. StatBite: FDA oncology drug product approvals in 2009. J Natl Cancer Inst 2010;102:219.

43. Chiew GGY, Wei N, Sultania S, et al. Bioengineered three-dimensional co-culture of cancer cells and endothelial cells: A model system for dual analysis of tumor growth and angiogenesis. Biotechnol Bioeng 2017;114:1865-77. 